\author{
EVS24 \\ Stavanger, Norway, May 13-16, 2009
}

\title{
Power bus control for series hybrid heavy-duty vehicles
}

\author{
Jukka Halme $^{1}$, Matti Liukkonen ${ }^{2}$, Matti Heiska ${ }^{3}$, Jussi Suomela ${ }^{1}$ \\ ${ }^{1}$ Helsinki University of Technology, TKK Department of automation technology, Otaniementie 17, P.O. Box 5500, \\ 02015 TKK, johalme@cc.hut.fi \\ ${ }^{2}$ Helsinki University of Technology, TKK Department of electrical engineering, Power electronics \\ ${ }^{3}$ Helsinki University of Technology, TKK Department of Engineering design and production, Vehicle engineering
}

\begin{abstract}
Heavy-duty vehicles OEMs have an increasing interest in alternative power train solutions. To gain benefits of hybrid power train, proper power bus control must be implemented. Control strategy refers to means necessary to meet duty cycle power demands while maintaining vehicle performance. Cascading controlling strategy division is proposed. Control method layering proposition is presented and control methods are divided into two categories, reactive and predictive methods. Finally these methods are implemented in series hybrid energy system model. Implemented reactive methods are load-based control, voltage-based control, voltage/load hybrid control and peak shaving control. Load data is measured data from electrical power train straddle carrier. One vehicle state predictive control method is implemented. Results are evaluated and discussed.
\end{abstract}

Keywords: Series hybrid, heavy duty vehicle, power control strategy, HEV

\section{Introduction}

Heavy duty off-road vehicle manufacturers are becoming more interested in alternative power train solutions mainly for the same reasons that car manufacturers have. Increasing running costs due oil price trends, tightening emission regulations and in some cases, direct reduction of emissions themselves. General drive cycles can be used to evaluate performance of hybrid passenger cars. Such generalization cannot be done in case of a off-road heavy duty vehicle. Each heavy duty vehicle has its own operating environment and duty cycle and thus hybridization for each vehicle has to be approached differently. However it is necessary to establish common overall theory and to find out basic control strategies that can be a starting point in designing control strategy for specific offroad heavy duty vehicle.

In this paper cascading controlling strategy division is proposed. Control method layering proposition is presented and control methods are divided into two categories, reactive and predictive methods. Finally these methods are implemented in series hybrid energy system model. Implemented reactive methods are load-based control, voltage-based control, voltage/load hybrid control and peak shaving control. Predictive method based on vehicle state is presented and implemented. Load data is measured data from electrical power train straddle carrier. This duty cycle data is used in simulating system performance using different control schemas. Results are then evaluated and discussed. 


\subsection{Energy management principals}

Hybrid systems are a replacement for conventional systems. This means that hybrid systems inherit performance requirements of conventional systems. In addition it is desirable that hybrid system can somehow exceed performance of conventional system in one or more aspects. Different aspects might be for example reduced fuel consumption, better performance or reduced emissions. Clear objective is essential when designing energy control strategies for hybrid systems.

\subsubsection{Hierarchy of needs}

Power bus has a hierarchy of needs. Each level of top down hierarchy objectives must be met before lower level objectives can be achieved. Figure 1 presents basic hierarchy of needs for series hybrid power system. Failure to keep voltage in power bus above minimum voltage required leads to collapse of power system. If primary objective can be reached, secondary objective in the hierarchy can focused upon. Vehicle performance objective means that the vehicle must be able to carry out its task with reasonable efficiency. Third object in hierarchy of needs is the criteria subject to optimization. Subject of optimization might be fuel consumption, emissions or some other criteria.

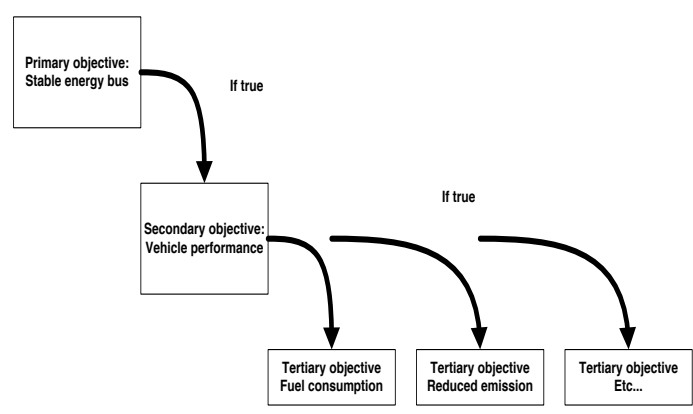

Figure 1: Power bus hierarchy of needs

\subsubsection{Control method layering}

Control methods can divided into three layers, strategic layer, power system layer and device layer as presented in figure 2. Layers are by no means exclusive but are bottom up inclusive.

Device layer represents decentralized control based on hardware. Device layer controls use measurable data as an input signal and thus do not require complex calculations in controller.
Controllers in this layer can include offline programmable control devices. For example one object in this layer could be voltage control in DC/DC-converter. Hardware based control results in best achievable response time but system controllability is more difficult to achieve. Time frame of control loop is near hardware's switching frequency.

Power system layer represents centralized software based control. Measurements are input signals and calculated data is output to actuators. Centralized control enables more complex control strategies and better overall system controllability. Minimum response time for software control is information bus delays plus calculation processing time.

Strategic is a control layer that can include data from other sources than the actual hybrid vehicle system. For example abstract control layer could include navigational information or other information external to power bus in its decision algorithms.

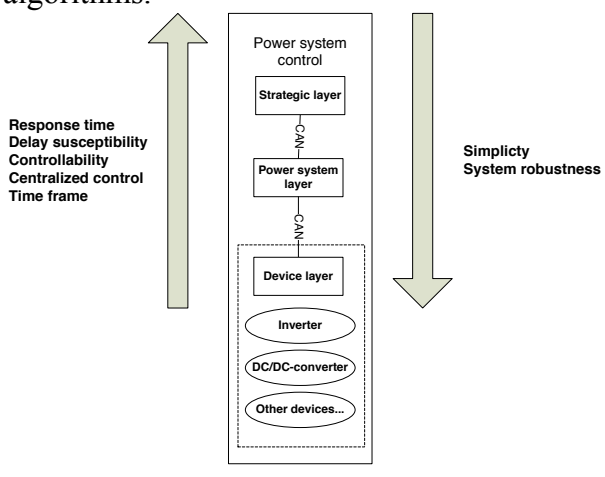

Figure 2: Control method layers

\section{Reactive and predictive control methods}

Reactive methods are control methods where control system reacts to changes in the power system and reacts to these circumstances in preprogrammed way. Reactive methods are categorized in device layer and power system layers.

Predictive methods are control methods where control system tries to estimate future in some way. Predictions might be cast about state of energy system and thus these methods are close to traditional predictive control methods in control theory and belong to either device or power system layer. Predictions might also be cast on a strategic 
layer. At this case energy system prepares to meet requirements of future load conditions. External data such as surface curve or gps data could be used. With this data energy system can be controlled in a way so that it is able to deliver required power during steep slopes or use slightly excessive power form energy storages during flat surface curve if regenerative energy can be predicted. Predictive control is one step further towards better overall system efficiency. Predictions can be cast about vehicles next task on its duty cycle. As an example while straddle carrier is moving without container, controller can assume that high power is needed in the near future for lifting the container. This way controller can keep certain amount of power in reserve for near future lifting task. Ultimately energy management can be taken to near optimal level if future duty cycle, container weights, distances between containers and surface curve is known.

\subsection{Reactive methods}

Regardless of control strategy method, DC-bus regulation is required as stated in figure 1 and it is underlying boundary condition in all control strategies. These control methods are placed on tertiary level of hierarchy of needs and software control layer in system control schema. Methods introduced below are all reactive control methods.

\subsubsection{DC-bus voltage control}

Voltage of DC-bus is measured and compared to reference value and proper action is taken. Voltage based control requires implementation of voltage hysteresis limits because DC-bus voltage is rarely entirely stable. Lack of hysteresis limits results in unwanted DC-bus voltage oscillation. Basic control logic is implemented so that when DC-link voltage is greater than voltage high hysteresis limit, energy storage is charged and respectively energy storage is discharged when DC-link voltage is below voltage low hysteresis limit. Voltage control requires high frequency control loop so this control method should be implemented in device layer.

\subsubsection{Load Control}

In load-based control it is assumed that required system power is known. Energy storage is charged and discharged in proportion to system load.

\subsubsection{Peak shaving control}

In peak shaving control energy storage discharge is activated when specific reference variable exceeds set value. Energy storage charge must be activated by some other control logic. For example basic setup for charge logic could be to charge whenever regenerative energy is available. Reference variable can be chosen freely but is commonly voltage, power or load. In the context of this paper, peak shaving control is load control based. Basically peak shaving control is identical to load based control with the exception that peak shaving control is triggered at higher power demand level and two energy storage DC/DC-converters operate in parallel state blocks.

\subsubsection{Hybrid control}

Mixing different control logics can potentially lead to better overall performance of energy system. In this paper hybrid control is defined as any combination of different control logics. Hybrid control implementation is composed of load control and voltage control. Each state transition condition is a combination of load condition and dc-bus voltage conditions.

\subsection{Vehicle state predictive method}

Some heavy-duty vehicles operate in structured environment where previous duty cycle resembles future duty cycles. This makes it possible to take novel approach to energy system control. In case of autonomous or semiautonomous vehicles the duty cycle is preplanned and therefore all actions are known beforehand. These systems are already in market [1].

Repetitive duty cycles effectively means that certain sub-cycles are repeated in predetermined sequence. Duty cycle division into sub-cycles offers distinct possibilities to more accurate energy system control during specific sub-cycle. It can be assumed that more information system designer has about duty cycle, more accurate predictions can be made about energy consumption during each sub-cycle and therefore about whole duty cycle.

This principle is presented below in context of straddle carrier carrying containers weighing up to 25 metric tons. Straddle carrier duty cycle division into sub-cycles is presented in figure 3. Duty cycle is divided into five sub-cycles, Initialization, Move to container, Lift container, Move to drop-off point and Drop container. These sub-cycles are shown 
as boxes in figure 3. Each of these sub-cycles includes different actions that are represented as diamond shaped boxes. Regenerative energy actions are lined with green and energy consuming actions are lined with red.

\subsubsection{Initialization}

Task parameters for next shift are calculated.

\subsubsection{Move to container}

Straddle carrier is driven to container. In this state it assumed that large transient power demand has to be met at Lift container -state. Accelerations and drive is supported by energy storages. Regenerative energy is harvested during braking. Main consideration of energy usage is in storing enough energy to assist lift of container in next sub-cycle. Energy required to lift the container can be calculated from harbor logs where weights and position of each container is maintained.

Routing energy through storages is not necessarily optimal energy usage because of the inevitable energy losses due transformations. In certain cases it is possible to use hoist itself as energy storage. This could be done by scheduling hoist lift to happen simultaneously with braking. In this way regenerative energy would be immediately used to lift hoist. This energy could be then used in accelerations by lowering hoist. Additionally this approach enables downsizing of energy buffers. This aspect of energy optimization is not studied in this paper but is subject to further research.

\subsubsection{Lift container}

This sub-cycle is most demanding in regard of energy consumption. Heavy transient power requirements have to be met. It is essential that energy storages have high enough state of charge at this sub-cycle to be able to assist energy system through whole sub-cycle.

\subsubsection{Move to drop-off point}

Straddle carrier moves to container drop-off point. Drive and acceleration modes are assisted as much as possible with remaining energy in energy storages. Regenerative energy from braking is harvested. Ideal situation would be that during this sub-cycle energy storages are depleted to minimum state of charge possible because in next sub-cycle it is assumed that large amount of regenerative energy is present. More accurate information about height and location of the drop-off point can be used to calculate amount of regenerative energy available during drop-off. This is the least amount of energy should be used in this sub-cycle.

\subsubsection{Drop container}

In this sub-cycle container is dropped to its desired location. Lowering hoist with container presents large transient regenerative energy source. State of charge of the energy storages must be sufficiently depleted to be able to harvest this regenerative energy.

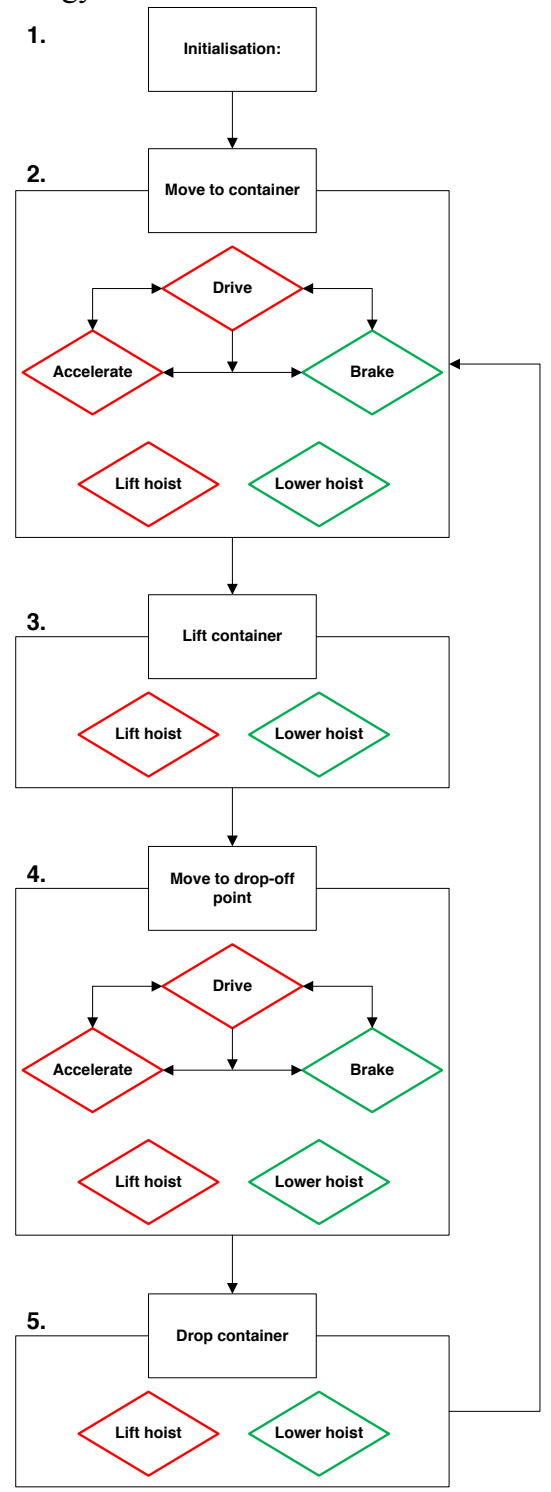

Figure 3: Rudimentary presentation of straddle carrier duty cycle 


\subsection{Series hybrid energy system model}

Model $[1,2]$ is constructed using Simulink basic library and SimPowerSystems library. Additionally energy system control logic is implemented using Stateflow library. Model represents series hybrid energy system on functional level. Switching frequency phenomena is ignored.

Model consists of four 250 volt 15.75 farad supercapacitors. Supercapacitors are divided into two sets, each with two series connected supercapacitors. Both sets have their own current controlled DC/DC-converter. Gen-set consists of single speed diesel engine that produces power for $200 \mathrm{~kW}$ generator. DC-link is modeled as a $\mathrm{RC}$-circuit. Brake chopper block is modeled as a large, set value voltage triggered resistor. Loadblock includes measured data from electrical power train straddle carrier. Model includes measured efficiency data for DCDC-converters and supercapacitors.

Control logic is implemented using Stateflow finite state machine. State transitions conditions are based on system load, DC-link voltage or combination of these two. State actions are direction of energy flow and requested current value from energy storage. Control system is assumed to have constant delay of 20 milliseconds. This delay represents accumulated delay of data transfers and data processing. Main sources of delays in real world applications are measurements, data transfers (via CAN-bus, Flex-ray ...) and data processing.

\subsubsection{Simulation targets}

Simulation objectives and constraints for simulation are defined as follows

1. DC-link voltage is $594+-50 \mathrm{~V}$

2. Vehicle is able to complete its duty cycle

3. Energy produced by diesel engine is minimized

4. Energy independency

DC-link stability is the most profound target in simulations. Voltages of +-50 volts are acceptable. Brake chopper is triggered at 680 volts to prevent potential hardware failures. If DC-link voltage is genuinely under 544 volts, simulation results are discarded as the situation is interpreted as DC-link collapse which compromises system performance, simulation target 2 .

Energy produced by diesel is the main numerical criteria subject of optimization. Focus is indeed in energy produced by diesel not energy consumed to produce. Diesel engine is assumed to operate in optimal efficiency range.

Fourth target means that energy system is selfsufficient in regard of the duty cycle. In practice this means that voltage level of supercapacitors at the end of duty cycle must be within $1 \%$ of the initial voltage level.

\subsection{Load data and simulation parameters}

Load data used in simulations is presented in figure 4. Relevant parameters are presented in table 1 .

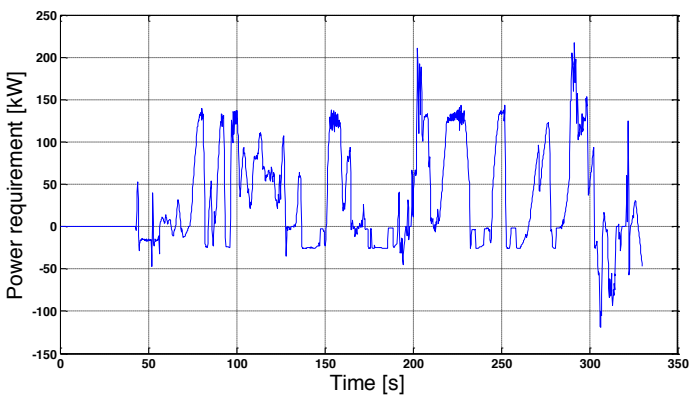

Figure 4: Load data of simulations

Table 1: Relevant model parameters

\begin{tabular}{|l|l|}
\hline Time step [s] & 0.0005 \\
\hline Signal delay [s] & 0.02 \\
\hline DC-link reference voltage [V] & 594 \\
\hline Super capacitor current limit [A] & 200 \\
\hline Super capacitor capacitance [F] & 15.5 \\
\hline Super capacitor voltage limits [V] & $250-500$ \\
\hline Brake chopper trigger voltage [V] & 680 \\
\hline
\end{tabular}

\section{Results}

\subsection{Performance evaluation of control methods}

Performance of control methods are evaluated by using following key ratios:

$E_{d}$ Energy produced by diesel during duty cycle [kWs] 
$E_{B C}$ Energy consumed in brake chopper during duty cycle $[\mathrm{kWs}]$

$E_{d} L_{\text {pos }}$ Ratio of energy produced by diesel and positive load during duty cycle

$E_{d} L_{\text {pos }}$ Returns value between 0 and 1 . This ratio has a value of one if whole energy requirement is produced by diesel engine. Thus smaller the ratio is more energy storages are used during the duty cycle.

$E_{d} L_{t o t}$ Is ratio between energy produced by diesel engine and total load. Total load consists of negative and positive values of load. This ratio returns value greater than or equal to one. Optimal value for this ratio is one. This represents circumstances where all available regenerative energy during duty cycle could be stored.
$P_{d, a v e}$ Average power produced by diesel during duty cycle $[\mathrm{kW}]$

$P_{d, \max }$ Maximum power produced by diesel during duty cycle $[\mathrm{kW}]$

Key ratios and simulation results are presented in table 2. Graphs of the simulations are included in appendices.

Table 2: Simulation results and key ratios of control methods

\begin{tabular}{|l|c|c|c|c|c|}
\hline & Load control & Voltage control & Peak shaving & Hybrid control & Predictive control \\
\hline $\boldsymbol{E}_{\boldsymbol{d}}[\mathrm{kWs}]$ & 10894 & 11178 & 10817 & 9809 & 10336 \\
\hline $\boldsymbol{E}_{\boldsymbol{B C}}[\mathrm{kWs}]$ & 0 & 146.85 & 32.79 & 16.50 & 0 \\
\hline $\boldsymbol{E}_{\boldsymbol{d}} \boldsymbol{L}_{\text {pos }}$ & 0.92 & 0.94 & 0.89 & 0.83 & 0.87 \\
\hline $\boldsymbol{E}_{\boldsymbol{d}} \boldsymbol{L}_{\text {tot }}$ & 1.13 & 1.16 & 1.12 & 1.01 & 1.07 \\
\hline $\boldsymbol{P}_{\boldsymbol{d}, \text { ave }}[\mathrm{kW}]$ & 33.01 & 33.87 & 32.78 & 29.73 & 31.32 \\
\hline $\boldsymbol{P}_{\boldsymbol{d} \text { max }}[\mathrm{kW}]$ & 119 & 142 & 132 & 123 & 130 \\
\hline
\end{tabular}

\section{Analysis}

As we can see from results presented in table 2, hybrid control results in best key values and ratios. Energy produced by diesel and average power required from diesel engine has smallest values. EdLpos value of 0.83 indicates that hybrid control maximizes usage of energy storages. EdLpos ratio indicates that $83 \%$ of energy required during duty cycle was produced by ICE. In regard of energy efficiency for example EdLtot value of 1.01 indicates that only 1 percent of regenerative energy could not be harvested. So hybrid control method is about 1 $\%$ far from optimum energy efficiency in this particular duty cycle.

Load control and predictive control had best performance in maintaining DC-link stability. These control methods had least amount of voltage crossovers beyond desired voltage sector
$V_{d c-\text { link ref }}=594 \pm 50 \quad$ volts. All control methods could keep up with main simulation target except voltage control. Figure 5 presents DC-link voltage in voltage control simulation in time interval $t=200 \ldots 300$ seconds. It can be observed that DC-link voltage collapses when $t=203$ and $t=290$ seconds. 


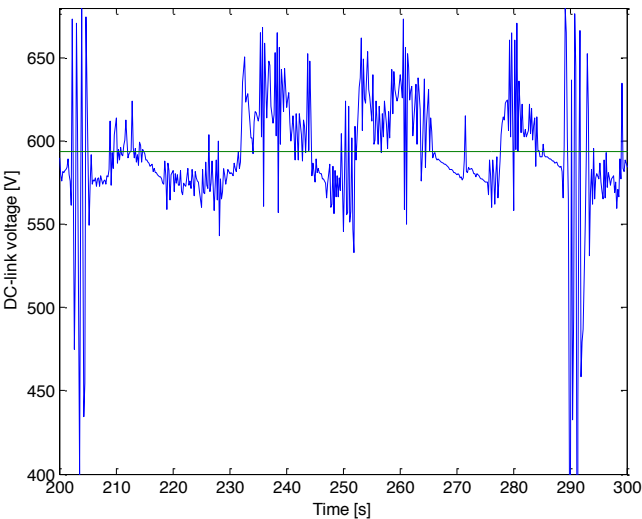

Figure 5: Voltage control $\mathrm{t}=200 \ldots 300 \mathrm{DC}$-link voltages, Delay $=20 \mathrm{~ms}$

DC-link voltage changes rapidly when energy system is under strain. It can be assumed that DC-link voltage has different values in each simulation time step, $T s=0.0005$ seconds. Energy system control delay is 20 milliseconds. This means that control system gets new data for processing once every fortieth time step. While it seems that voltage control method would be valid and well suited for generalization, it is found out to be very susceptible to delays. Rapidly changing process with huge I/O delays means that if voltage control is to be applied, necessary actions must be taken to counter effect of delays in system. Figure 6 presents voltage control case with a system delay of 10 milliseconds and figure 7 with a system delay of 1 millisecond. It can be observed that delay indeed has significant effect especially in voltage control method. In 10 milliseconds case, voltage drop is 120 volts while in 20 milliseconds case voltage drop was well over 200 volts. In 1 millisecond case voltage drop was only little over 50 volts which, as defined in simulation targets, is acceptable result. This leads to a conclusion that voltage control should implemented only in device layer.

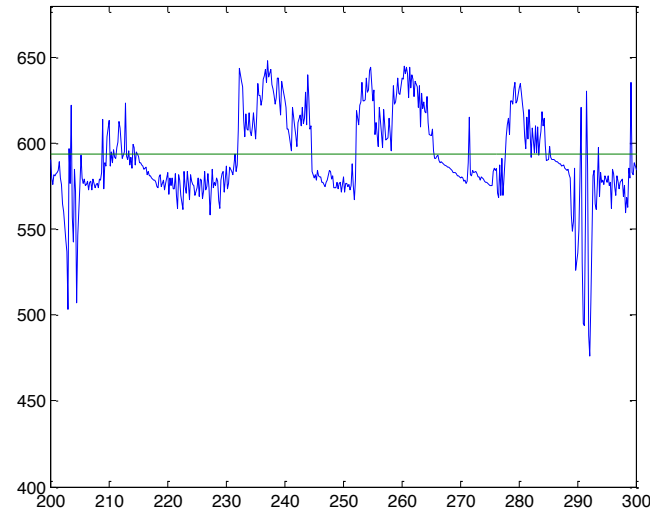

Figure 6: Voltage control $t=200 \ldots 300$ DC-link voltages, Delay $=10 \mathrm{~ms}$

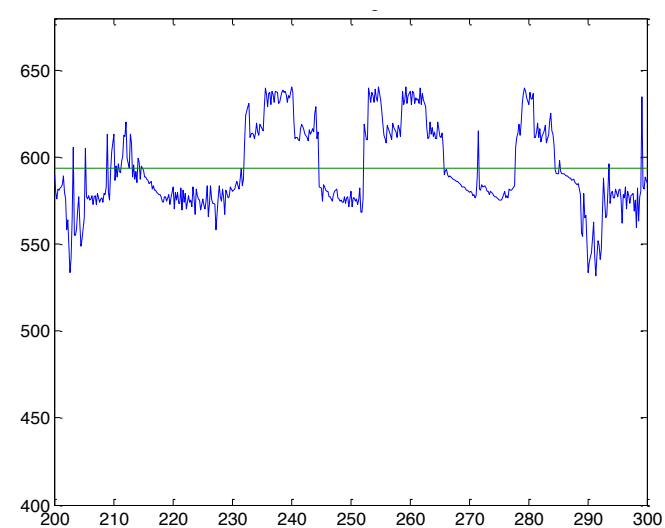

Figure 7: Voltage control $t=200 \ldots 300$ DC-link voltages, Delay $=1 \mathrm{~ms}$

Hybrid control method came up with best results with this duty cycle. Basically hybrid control schema combines voltage control and load control state transition conditions. Thus it is made certain that discharge and charge events take place at proper time.

It seems that intuition, more information leads to better decisions, is true also in power bus management. However there certainly is a limit how much information can be used effectively in purely reactive control. One possible approach is to use other information than power bus measurements in control logics. Information external to power system is used in vehicle state predictive control. In this paper cyclic control schema based on actual duty cycle was implemented. Division into sub-cycles makes energy management easier to optimize. In rulebased control schemas plenty of different possible situations must be taken into account. By dividing duty cycle into sub-cycles own distinct control 
rules can be designed for each sub-cycle. In purely rule-based control there exist redundant rules in regard of some certain sub-cycle. In best case scenario these rules are just redundant in regard of some sub-cycles. Ill-defined rule set might lead to suboptimal performance in some sub-cycles or in the worst case scenario these redundant rules can have detrimental effects on energy efficiency.

By using duty cycle division most of these drawback can be countered. For each sub-cycle, own rule set can be defined so that energy efficiency for the whole duty cycle can be improved. Additionally when sub-cycles have their own distinct character, design of the rule set is much easier if only those distinct characteristics have be taken into consideration without having to think about effects of these rules in some other phase of duty cycle.

While key ratios for predictive control were not better than hybrid control's ratios, it can be assumed that vehicle state predictive has much potential in it and it should be subject further research. Most noticeable aspect of this particular predictive control method is that is more easily to generalize than other rule sets. Duty cycles resemble each other in macro level but variation in travelled distances and container weights makes strict rule sets harder to define so that they operate in optimal way in every situation.

\section{Acknowledgments}

This paper is part of an ongoing project $H Y B L A B$, funded by the Multidisciplinary Institute of Digitalization and Energy $(M I D E)$ of Helsinki University of Technology TKK.

\section{References}

[1] J.Halme, Energy bus management in series hybrid system, M.Sc. (eng) thesis 2008

[2] M. Liukkonen, Functional Simulations of Power Electronics Components in Hybrid Machinery, M. Sc. (eng) thesis 2008, http://lib.tkk.fi/Dipl/2008/urn012135.pdf

[3] H. Durrant-Whyte, An autonomous straddle carrier for movement of shipping containers, IEEE Robotics and Automation magazine vol 14, 2007, DOI: Digital Object Identifier 10.1109/M-RA.2007.901316

\section{Authors}

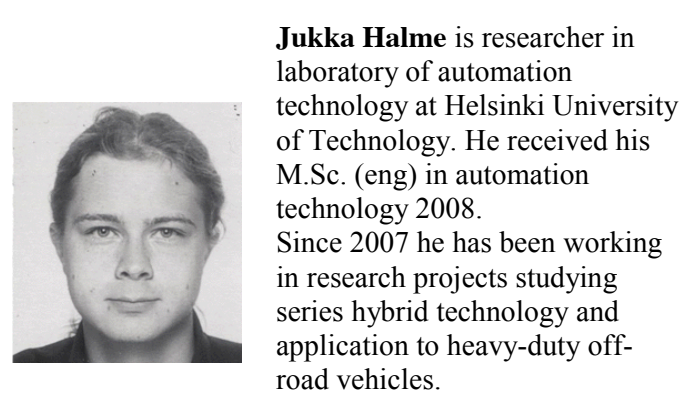

Matti Liukkonen is an undergraduate with nearly finished M.Sc. (Eng.) degree in electrical engineering from the Helsinki University of Technology (HUT), Espoo, Finland.

In 2008, he wrote his master's thesis from "Functional

Simulations of Power Electronics Components in Hybrid

Machinery".

Since 2007, he has been working as research assistant in a project

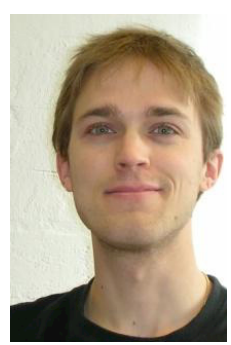
related to simulation and control of series hybrid power transfer in HUT.

From 2005 to 2007, he was working as a research assistant in R\&D department in ABB, Drives Oy, Finland, and also in Corporate Research, ABB Switzerland Ltd. 



Matti Heiska received his M.Sc. degree in Vehicle Engineering from Helsinki University of Technology (TKK), Finland, in 2002. During 2002-2008 he worked in Patria Land \& Armament as a R\&D Engineer. Since 2008 he has been working as a Research Scientist in Department of Automation and Systems Technology in TKK. His main research interests are in the field of vehicle control systems and hybrid electric vehicle technologies.

Jussi Suomela is senior research scientist and project manager in Department of Automation and Systems Technology in Helsinki University of Technology TKK since 1992. His main research areas are hybrid electric vehicles and field and service robotics. $\mathrm{He}$ received his doctoral degree from TKK in 2004. 


\section{Appendices:}

\subsection{Simulation results}
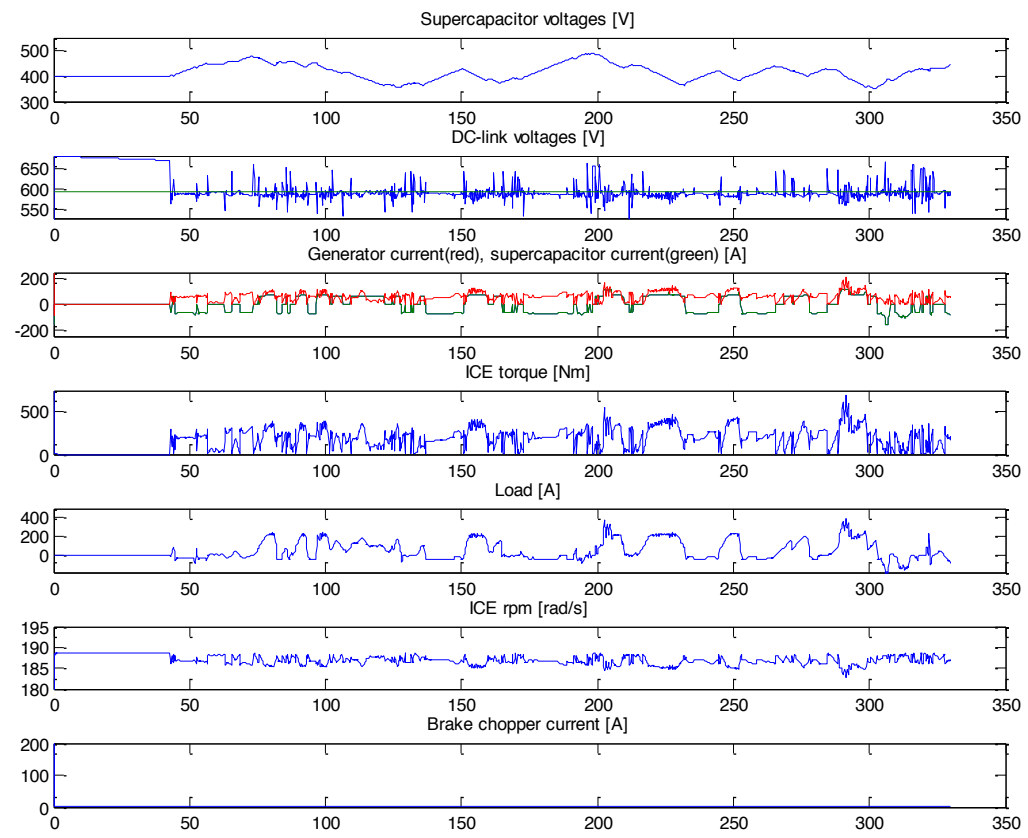

Figure 8: Load control simulation results
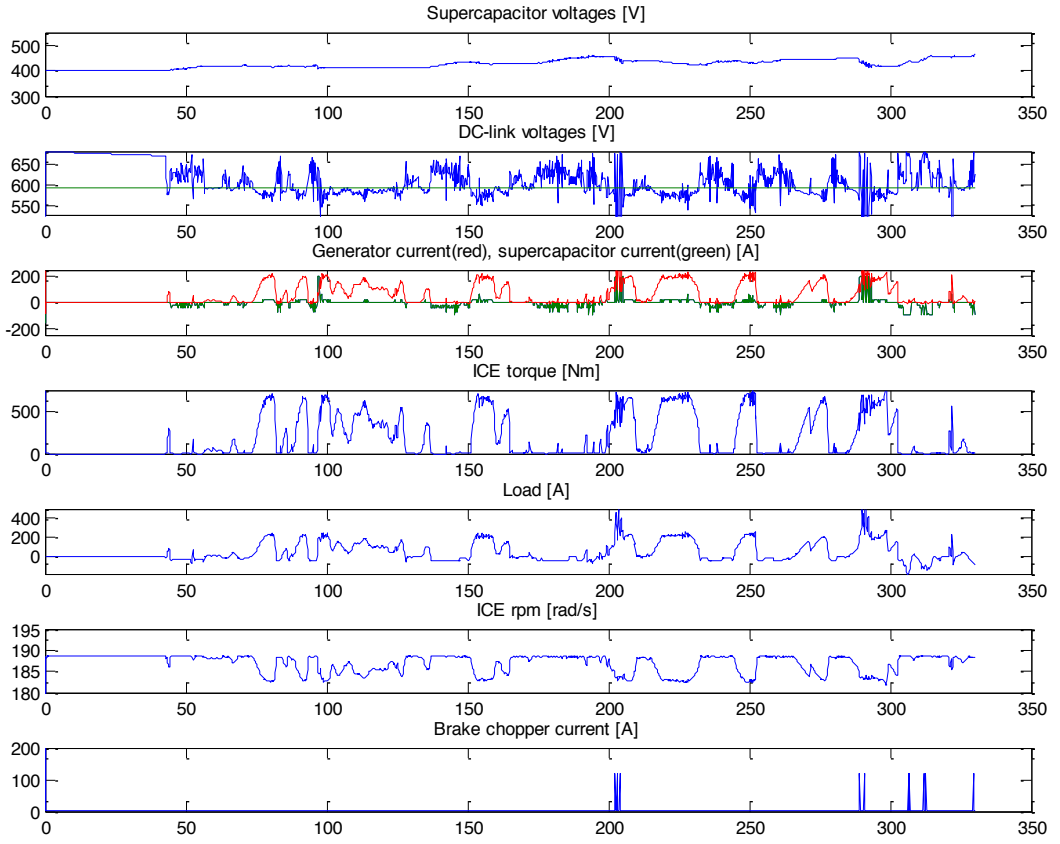

Figure 9: Voltage control simulation results 

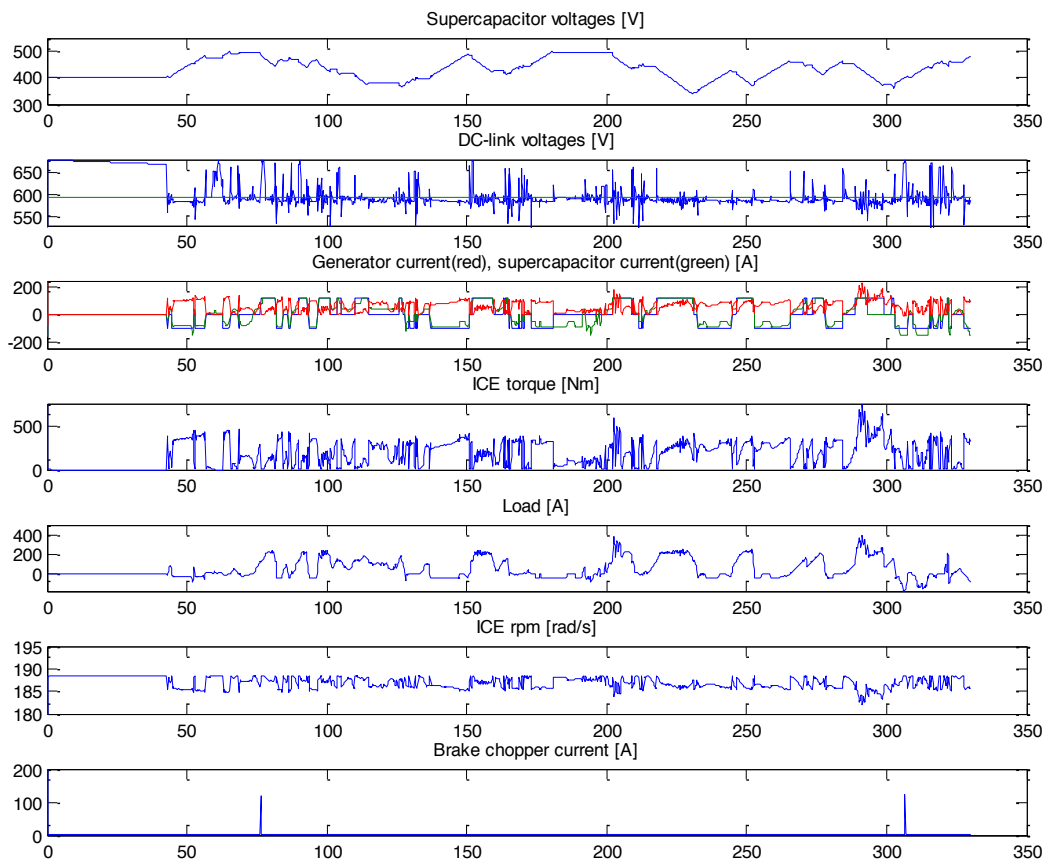

Figure 10: Peak shaving control simulation results
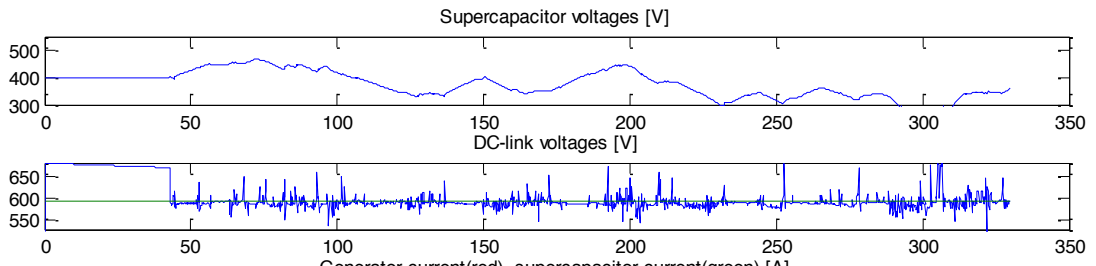

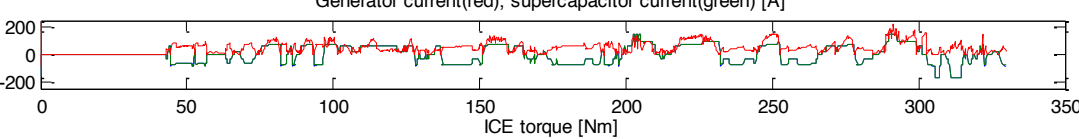
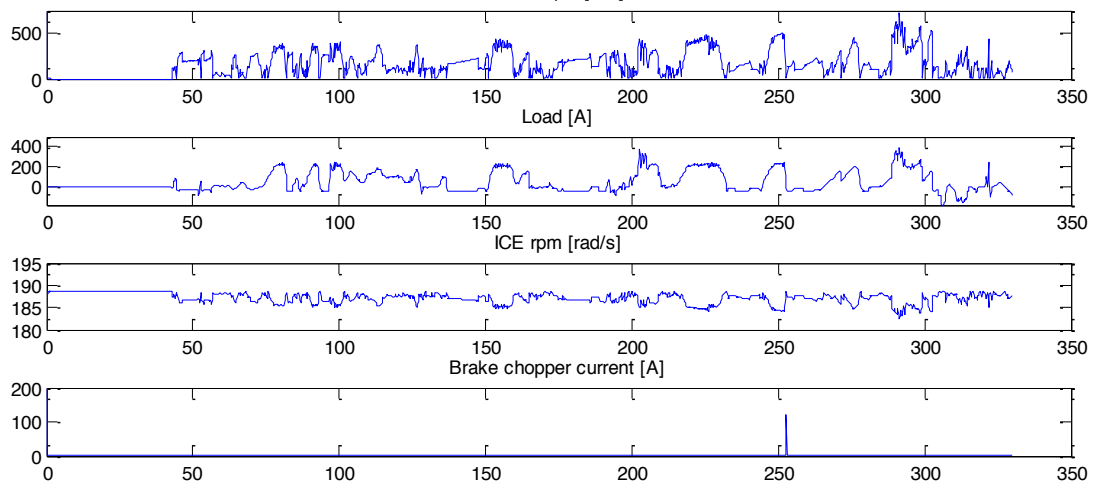

Figure 11: Hybrid control simulation results 


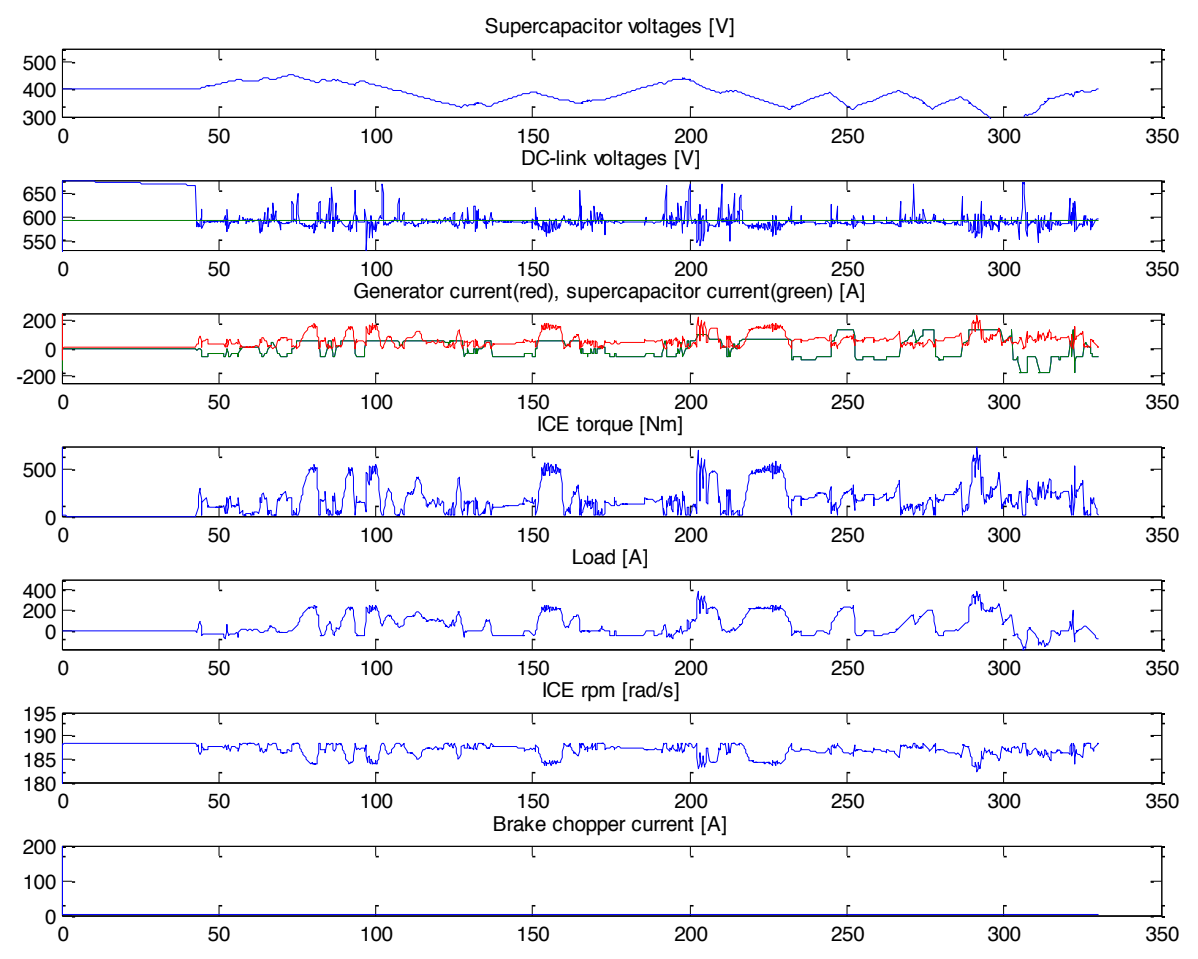

Figure 12: Vehicle state predictive control simulation results 